\title{
Consideration of Tool Chamfer for Realistic Application of the Incremental Hole-Drilling Method
}

\author{
Nicola Simon ${ }^{1, a^{*}}$, Jens Gibmeier ${ }^{1, b}$ \\ ${ }^{1}$ Karlsruhe Institute of Technology (KIT), Institute for Applied Materials (IAM), Kaiserstr. 12, D- \\ 76131 Karlsruhe, Germany \\ anicola.simon@kit.edu, bjens.gibmeier@kit.edu
}

\begin{abstract}
Keywords: Residual Stress Measurement, Incremental Hole-Drilling Method, Hole Geometry, FEM
\end{abstract}

\begin{abstract}
The incremental hole-drilling method is well-established in residual stress analysis. A small blind hole is drilled incrementally and the strain relief is measured on the sample's surface. In order to calculate residual stresses from the measured strain calibration data is necessary. Typically, the calibration data is determined numerically and is based on the assumption of an ideal cylindrical blind hole. However, widely used six-blade milling bits have rather large chamfers at the cutting edges, which result in hole geometries that clearly differ from the ideal cylindrical blind hole. Especially in the first drilling increments a significant impact on the calibration data by the real hole geometry is expected. In this work, a numerical model is set up that allows for consideration of tool chamfers. A systematic finite element study is carried out to analyze the effect on relieved strains. Calibration data is computed for the ideal blind hole as well as for the realistic hole geometry. Finally, numerical results are compared with experimental results gained by defined uniaxial loading experiments. The results clearly indicate a significant impact of the tool chamfer geometry for strain relief and stress data close to the surface. Hence, based on the results it is highly recommended to consider the real tool geometry to provide accurate stress evaluation by means of the incremental hole-drilling method in particular for the first drilling increments.
\end{abstract}

\section{Introduction}

The incremental hole-drilling method is a widely used mechanical method for residual stress depth profiles analysis due to its simple instrumentation and fast execution. Since J. Mathar [1] first proposed the test method in 1933 it has been under constant development. State of the art is incremental drilling of blind holes of small diameters in order to determine local stress gradients. The method is based on redistribution of residual stresses due to local material removal. Entailed strain relaxations can be measured on the surface area around the hole e.g. by means of strain gages. Due to the fact that strains are only partly released when introducing a blind hole, calibration data is needed to evaluate the residual stress profile from measured strains. Usually, finite element (FE) simulations are used to calculate these calibration data. In commercially available evaluation routines generally an ideal cylindrical blind hole is considered. Flaman [2] showed that the experimental setup of high speed drilling in combination with inverted cone end mills lead to the best approximation of the ideal cylindrical blind holes, while inducing a negligible amount of machining stresses. The widely used six-blade tungsten carbide milling bits with nominal diameters of 0.8 or $1.6 \mathrm{~mm}$, that are used in commercially available pneumatic high speed drilling devices, have large chamfers at the cutting edges, which result in hole geometries that differ from the ideal cylindrical blind hole.

Two shortcomings arise out of this geometrical deviation (see also Fig. 1):

- a remaining bottom fillet for each single drilling increment

- an increasing hole diameter for the first drilling increments 
In Scafidi, et al. [3] the first impact, i.e. the bottom fillet, was investigated by considering a bottom fillet radius in numerical simulations. It was figured out, that deviations of strain relaxations can cause erroneous results in case of large bottom fillet radii. In Nau, et al. [4] different kinds of end mills and their influence on the occurring hole geometries for a nominal hole diameter of $1.6 \mathrm{~mm}$ were studied. A numeric model was set up considering the effect of increasing hole diameters in the first drilling steps by using the mean hole diameter over the actual removed depth increment. The conclusion of both studies was that the consideration of more realistic tool geometries for calibration results in more meaningful stress data. However, in [3] and [4] only the general effects were discussed without taken into account the real chamfered cutting edge of the most often applied end mills. Furthermore, up to now only chamfered tools with a nominal diameter of $1.6 \mathrm{~mm}$ were considered.

In our project we have the inherent necessity to apply small hole diameters using a nominal tool diameter of $0.8 \mathrm{~mm}$ for residual stress depth distributions showing a relative steep gradient close to the surface. Hence, in this work a FE model is set up for a more accurate simulation of the hole geometry by considering the real chamfered edges using a conventional tapered end mill with $0.8 \mathrm{~mm}$ diameter. Thus, both shortcomings of the non-ideal cylindrical hole geometry are taken into account (remaining bottom fillet and unsteady hole diameter). The FE model can be used to calculate chamferconsidering calibration data for reliable stress
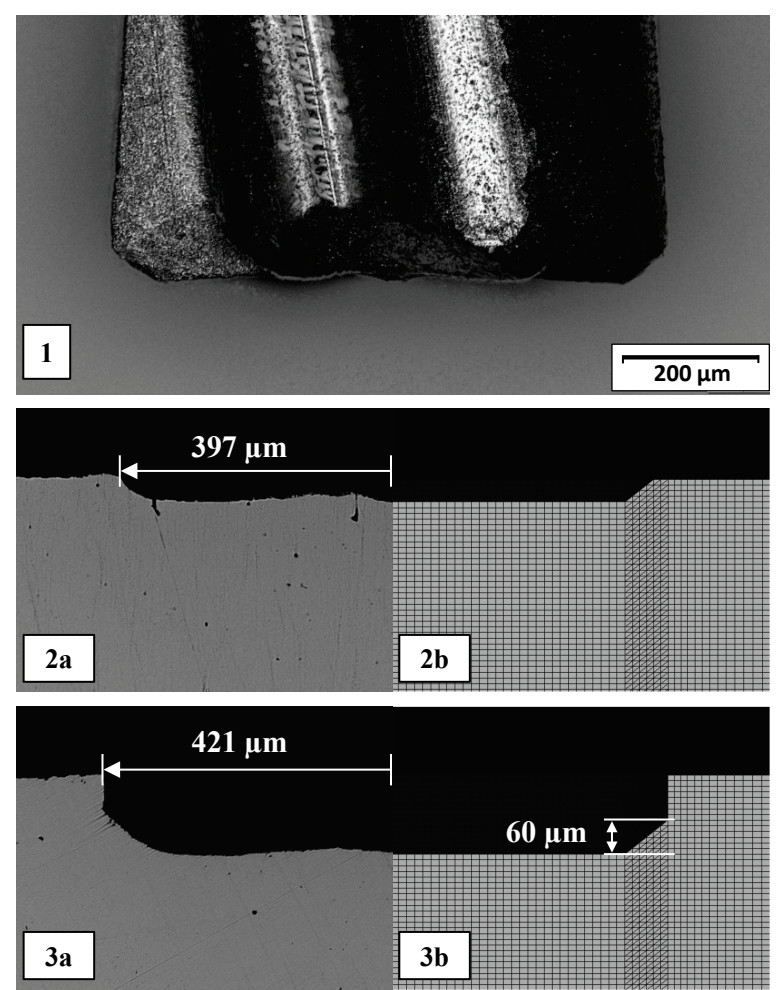

Fig. 1: Six-blade TiN coated tungsten carbide end mill with nominal diameter of $0.8 \mathrm{~mm}$ (1), drilled holes of $40 \mu \mathrm{m}$ depth (2) and $140 \mu \mathrm{m}$ depth (3), cross section of drilled sample (a), side view of FE model (b). evaluations using the differential method [5]. Finally, the numerical results are validated by experimental findings of a defined 4-point bending test.

\section{Finite element simulation}

Finite element (FE) model. The 3D FE model was defined in ABAQUS and consists of 750,000 elements of type C3D8R and C3D6. This hybrid element model is needed to take the chamfer geometry into account. An inverted cone tungsten carbide six-blade end mill of $0.8 \mathrm{~mm}$ diameter with a chamfer height of $0.06 \mathrm{~mm}$ at an angle of $45^{\circ}$ was assumed for the numeric model (Komet, Gebr. Brasseler GmbH \& Co. KG). Fig.1 shows the cutting edges of the end mill and the cross sections of a steel sample with two drilled blind holes of different depths. On the right hand side a side view of the FE model is presented. It can be seen that it is in good agreement with the actual blind hole geometry, even for a small drilling depth of $40 \mu \mathrm{m}$. Due to the symmetry of the calculated problem only a quarter model was used. A full view of the model is shown in Fig. 2. The drilling process is simulated by stepwise removing the elements in the region of the hole (drilling increments are highlighted in Fig. 2). For each drilling step the released strains on the surface area around the hole were calculated and averaged at element surface nodes. In a post processing step, the strains were integrated and averaged over three virtual strain gage areas at the positions $0^{\circ}, 45^{\circ}$ and $90^{\circ}$ leading to three single strain values $\varepsilon_{0^{\circ}}, \varepsilon_{45^{\circ}}, \varepsilon_{90^{\circ}}$, comparable to those, gained in experimental studies. 
Strain gage rosette dimensions considered in the model are similar to type A residual stress strain gage rosette according to the ASTM E837 [6].

For the hole-drilling simulation a biaxial nominal stress state of $\sigma_{\text {nom }}=\sigma_{\mathrm{x}}=\sigma_{\mathrm{y}}=300 \mathrm{MPa}$ was applied on the outside surfaces of the quarter model (see Fig. 2). Linear elastic material behavior of isotropic steel with a Young's modulus of $210 \mathrm{GPa}$ and a Poisson's ratio of 0.3 was applied. The chosen hole diameter of $0.8 \mathrm{~mm}$ is the minimum achievable hole diameter in practical application by the use of an end mill of the assumed size. Two identically meshed numeric models have been used to simulate the resulting strain data $\varepsilon_{\text {chamfered, for the chamfered drilled }}$ hole and $\varepsilon_{\text {cylindrical }}$, for the ideal cylindrical hole, respectively.

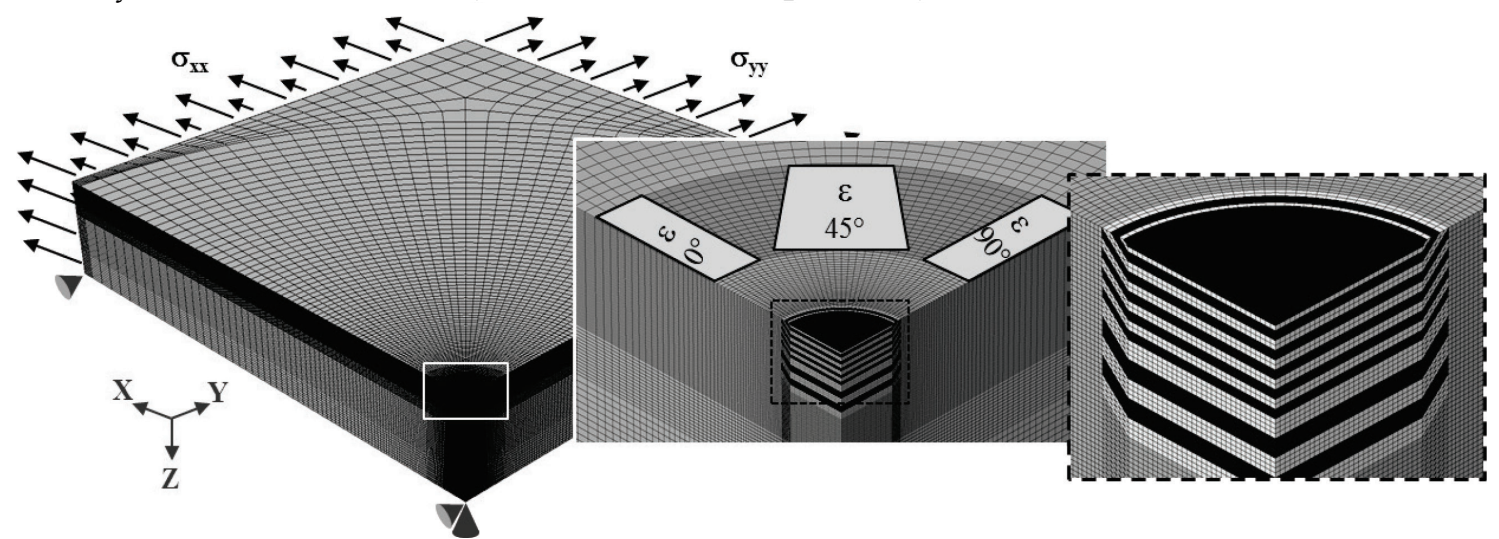

Fig. 2: ABAQUS 3D FE-model with detailed views of the position of the strain gages and the drilling increments.

Stress calculation was carried out for the strain data $\varepsilon_{\text {chamfered }}$ of the hole-drilling simulation considering chamfered holes, i.e. the better approximation of the real hole geometry. The stress was evaluated using calibration data $\mathrm{C}_{\text {cylindrical, }}$, which are typically provided by commercially available hole-drilling evaluation software, assuming ideal cylindrical holes. In comparison, a stress evaluation based on the consideration of the chamfer using the calibration data $\mathrm{C}_{\text {chamfered }}$ was carried out. Since we are most likely interested in the near surface data, the results are only presented up to a drilling depth of $0.4 \mathrm{~mm}$.

Results and discussion. In order to show the impact on measured strain relaxations, absolute and relative strain deviations (referred to the ideal hole geometry) between the two drilled hole geometries are shown in Fig. 3a. Due to the axi-symmetric stress state, only one strain gage component is plotted. Drilling steps with increasing hole diameters (up to $0.06 \mathrm{~mm}$ depth) are shaded in a grey zone. In general, for the loading state considered strain relaxation is higher for the assumption of an ideal blind hole. However, a significant decrease of the relative strain deviation can be seen. From $30 \%$ strain deviation in the first increment of $0.01 \mathrm{~mm}$, values are decreasing to less than $5 \%$ for the last drilling depth of $0.4 \mathrm{~mm}$. The absolute strain deviation is showing a maximum of $10 \mu \mathrm{m} / \mathrm{m}$ at about $0.24 \mathrm{~mm}$ drilling depth. This behavior can be explained by the interaction of two effects that are accompanied by the geometrical deviation of the drilled holes. The first effect is the remaining hole-bottom fillet, which is leading to a significant deviation of the removed material volume (see Fig. 3b). Especially for the first drilling increments, where the final hole diameter is not yet reached, the removed volume for the ideal hole geometry is 1.2 the size of the chamfered hole volume. This difference of volume clearly has an impact on the large relative strain deviation in the first drilling increments. Furthermore, the hole geometry influences the notch factor of the drilled hole (second effect that depends on the stress state considered), leading to a higher maximum stress in case of a sharp edged ideal cylindrical hole. From a drilling depth of $0.24 \mathrm{~mm}$ this effect is declining, leading to a decrease of the absolute strain deviation. It must be mentioned that in practical application, typically larger hole diameters occur due to slight asymmetric end mills or slightly eccentrically installed turbine shafts. Numeric simulations regarding larger hole diameters up to 
about $0.96 \mathrm{~mm}$ showed that larger hole diameters lead to smaller relative strain deviations for the same strain gage positions.

Thus the simulation with a drilled hole of $0.8 \mathrm{~mm}$ in diameter gives a limit of the maximal relative strain error that can occur by disregarding the chamfered hole geometry.

a)

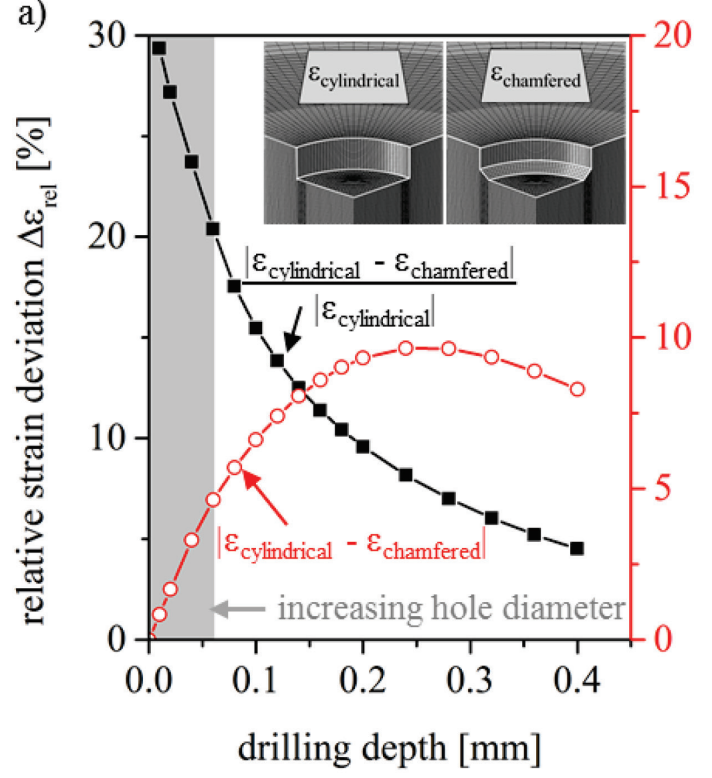

b)

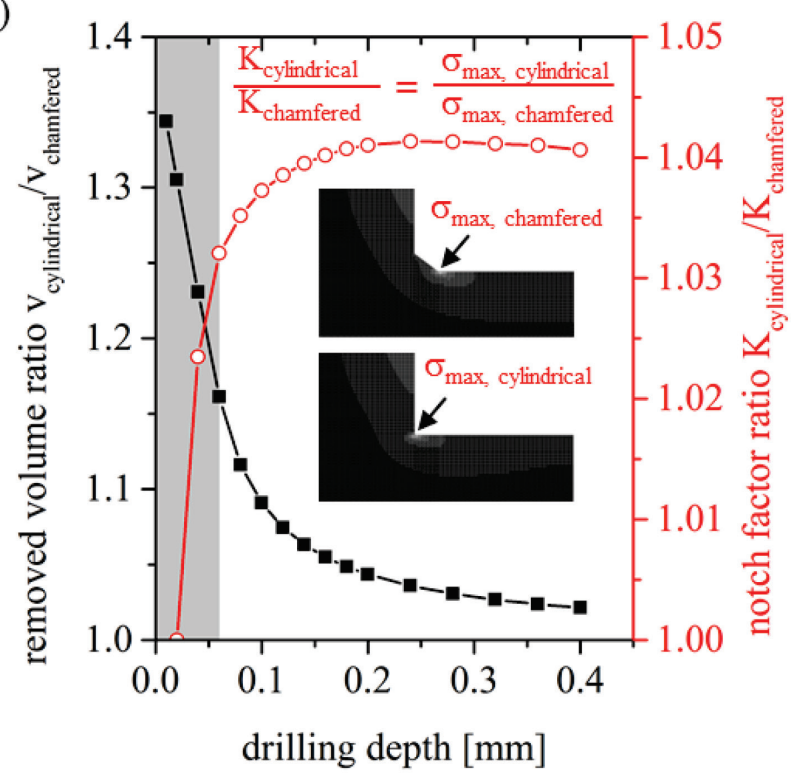

Fig. 3: strain deviation of $\varepsilon_{\text {cylindrical }}$ and $\varepsilon_{\text {chamfered }}(a)$, removed volume ratio and notch factor ratio (b).

The stress evaluation according to the differential approach of the chamfered hole-drilling simulation with calibration data considering a cylindrical hole is showing high stress deviations from the nominal stress (see Fig. 4). The large strain deviations in the first increments lead to a significant stress error of about $27 \%$ in maximum. In contrast, stress evaluation with calibration data considering the chamfer geometry leads to small stress deviations of less than $2 \%$, due to numerical errors. Following the numerical results, the use of chamfer-considering calibration data is highly recommended for an accurate stress evaluation.

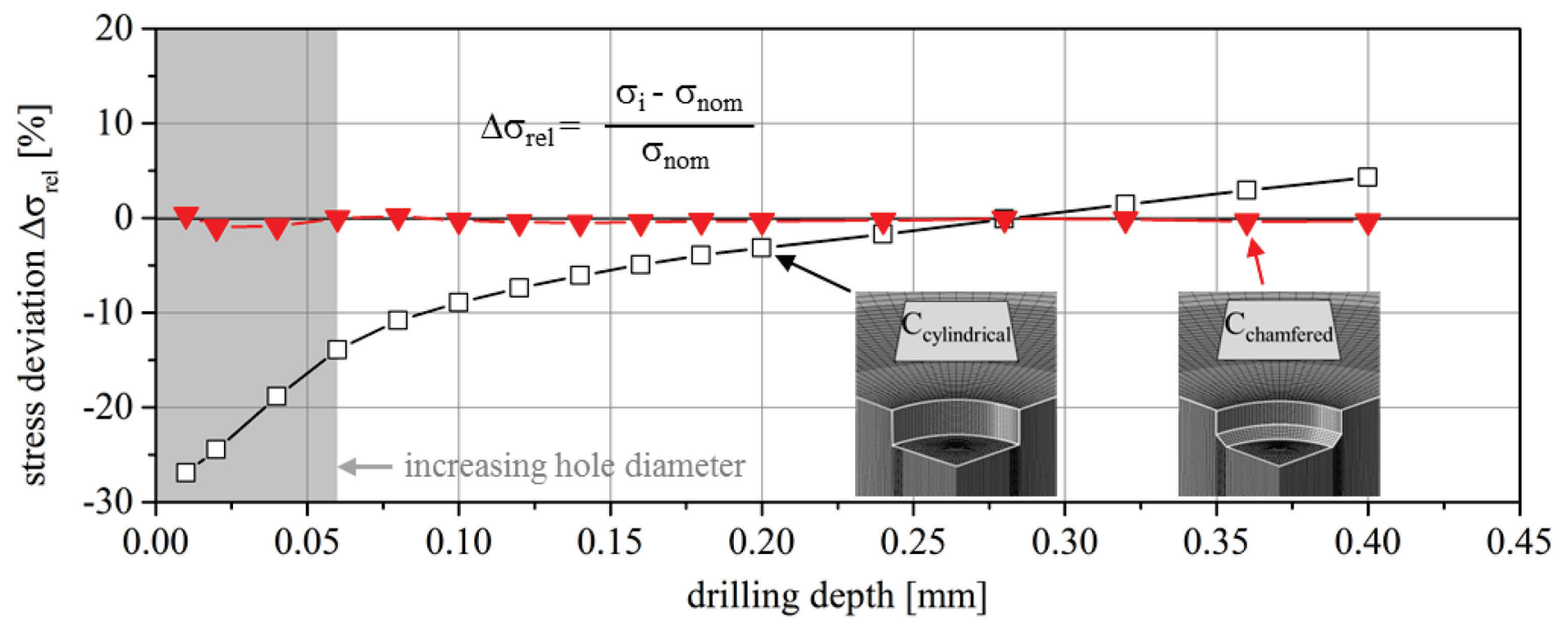

Fig 4: Stress evaluation of the chamfer-considering hole-drilling simulation with calibration data $C_{\text {chamfered }}$ and $C_{\text {cylindrical }}$. 


\section{Experimental application}

Experimental setup. Experimental tests were carried out on a fine grained construction steel S690QL bar with length $1=160 \mathrm{~mm}$, width $\mathrm{w}=40 \mathrm{~mm}$ and thickness $\mathrm{t}=6 \mathrm{~mm}$. A stress relief annealing $\left(1 \mathrm{~h}, 600^{\circ} \mathrm{C}\right)$ was conducted prior to the hole-drilling experiments. XRD residual stress analyses according to the $\sin ^{2} \psi$-method were carried out on the bottom and top surface after the stress relief treatment. Residual stress values were less than $13 \mathrm{MPa}$, hence the sample can be assumed to be stress' free'. The sample has been loaded by means of a four point bending device, with a distance of the inner supports of $50 \mathrm{~mm}$. A uniaxial bending stress gradient from $-400 \mathrm{MPa}$ on the top surface to $400 \mathrm{MPa}$ on the bottom surface over the bending height of $6 \mathrm{~mm}$ was applied. The load does not exceed more than about $58 \%$ of the materials minimum yield strength of $690 \mathrm{MPa}$. For the elastic material properties a Young's modulus E $=210 \mathrm{GPa}$ and a Poisson's ratio $v=0.3$ have been assumed. Three hole-drilling experiments have been carried out with measuring points in a distance of $10 \mathrm{~mm}$ to each other. After the three experiments each final hole diameter has been measured and was implemented exactly in the numeric model to calculate chamfer-considering calibration data. Finally the experimental strain data has been smoothed with cubic spline functions and the stress gradient has been evaluated by means of conventional calibration data $\mathrm{C}_{\text {cylindrical }}$ and by means of chamfer-considering calibration data $\mathrm{C}_{\text {chamfered, }}$, respectively. For presentation of the results the mean values of the 3 single experiments are used.

Results and discussion. Fig. 5 shows the evaluated stress gradients as a function of the drilling depth. The mean stress value of the three drilling experiments for each single depth increment is shown. Standard deviation of the mean stress value is given by surrounding error lines. Whereas the conventional approach (calibration data for cylindrical hole geometry) leads to a stress deviation of approx. $70 \mathrm{MPa}$ in the first increments, the calibration for considering the chamfer geometry leads to a stress value that is in good agreement with the nominal stress with deviation of less than $26 \mathrm{MPa}$. From a drilling depth of $0.08 \mathrm{~mm}$ on, the stress deviation of both evaluation methods is tolerable. Due to a slightly larger experimental hole diameter, the stress errors in the first increments do not exceed the large relative stress deviations of $30 \%$ that appear in the simulation for a hole diameter of $0.8 \mathrm{~mm}$

(Fig. 4).

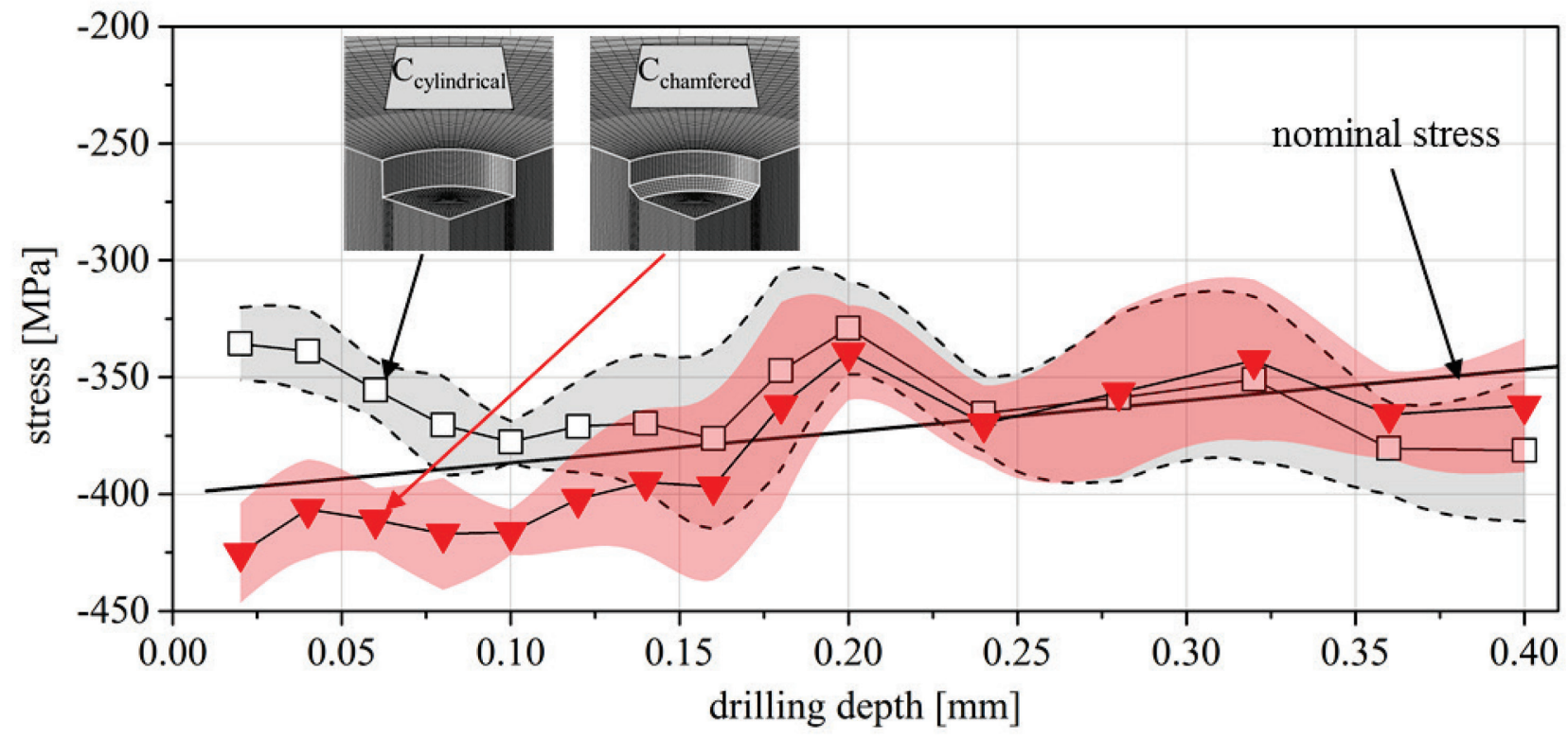

Fig. 5: Stress evaluation of the experimental loading test with calibration data $C_{\text {chamfered }}$ and $C_{c y l i n d r i c a l}$, mean stress values with standard deviations for the 3 drilling experiments.

It has to be taken into account that in experimental application strain measurement errors always appear due to scattering in the strain signal. However, for high strain relaxation in the first drilling 
increments, the absolute strain measurement errors are negligible. The data clearly show that the discrepancies indicated through the numerical simulations are also obvious for the near surface residual stress data determined by the defined uniaxial loading experiment. Due to the significant stress deviations in the surface near area the use of chamfer-considering calibration data is recommended, if this experimental setup is used and accurate stress values in the first increments are needed.

\section{Summary}

In this study, a FE model was set up that considers realistic tool chamfers for evaluation of residual stresses by means of the incremental hole-drilling method. The two effects of increasing hole diameter and a reduced material removal are taken into account. Strain data of the simulated holedrilling experiment for a hole diameter of $0.8 \mathrm{~mm}$ showed significant deviations to the conventional approach, based on ideal cylindrical hole geometry. Especially in the first increments, the increasing hole diameter due to chamfered end mill geometry is leading to large relative strain deviations. By use of conventional calibration data, erroneous stress data arise. Experimental hole-drilling tests have been carried out for a defined 4-point-bending loading test that confirmed the numerical findings. Chamfer-considering calibration data obtain a smaller stress deviation from nominal stress for the sensitive depth increments in the surface near area. Thus it can be concluded in the presence of large residual stresses in surface near areas, the chamfer-considering calibration is highly recommended to achieve accurate stress evaluation in particular for the first drilling increments.

\section{Acknowledgement}

The Graduate School 1483 "Process Chains in production: modeling, interactions and assessments of process zones" and the German research foundation (DFG) are gratefully acknowledged for funding.

\section{References}

[1] J. Matar, Determination of initial stresses by measuring the deformation around drilled holes, Transactions ASME Vol.56 No. 4 (1934) 249-254.

[2] M. Flaman, Brief investigation of induced drilling stresses in the center-hole method of residualstress measurement, Experimental Mechanics 22(1) (1982) 26-30. http://dx.doi.org/10.1007/BF02325700

[3] A. Nau, B. Scholtes, Evaluation of the high-speed drilling technique for the incremental holedrilling method, Experimental Mechanics 53 (2013) 531-542. http://dx.doi.org/10.1007/s11340012-9641-1

[4] M. Scafidi, E. Valentini, B. Zuccarello, Effect of the hole-bottom fillet radius on the residual stress analysis by the hole drilling method, ICRS- 8 The $8^{\text {th }}$ International Conference on Residual Stress - Denver (2008) 263-270.

[5] T. Schwarz, H. Kockelmann, Die Bohrlochmethode - ein für viele Anwendungsbereiche optimales Verfahren zur experimentellen Ermittlung von Eigenspannungen, Messtechnische Briefe 29 (1993) 324-333.

[6] ASTM, Determining residual stresses by the hole-drilling strain-gage method, ASTM Standard Test Method E837-13a (2013) 